I. D. Karachentsev

Special Astrophysical Observatory

Academy of Sciences, USSR

\title{
1. GENERAL REMARKS
}

During recent years the problem of the virial paradox has grown less acute for many galactic systems, especially for those having few members. As an example one can take the revision of virial mass-toluminosity ratio, $f=M / L$, for de Vaucouleurs' groups performed by Materne and Tammann (1974). The reduction of the f-estimate is due to various reasons: an increase in accuracy in measuring radial velocities of galaxies, especially noticeable for $21-\mathrm{cm}$ surveys of groups (Fisher and Tully 1975), regard for the sub-structure of systems of galaxies and improved exclusion of accidental members of groups (referred to here as "optical" members). Some very scattered systems proved to be low-contrast fluctuations of the expanding background, and not physical groups in their usual sense.

The most noteworthy recent feature has been the transition from episodic virial mass estimates for some selected systems, using data of different quality by different authors, to planned programmes of regular investigation of certain kinds of systems of galaxies. I would like to emphasize the following advantages of such an approach: a we11-defined selection criterion of systems allows one to estimate quantitatively selection effects and the presence of optical members in each sample; the uniformity of radial velocity measurements decreases the probability of errors encountered in heterogeneous compilations of data.

\section{PAIRS OF GALAXIES}

Being the simplest type of system, pairs are most suitable for investigation of the virial paradox. At our Observatory, in cooperation with other astronomers, a programme of systematic radial velocity measurements has been started for double galaxies. The catalogue of 603 isolated pairs by Karachentsev (1972) has been taken as a basis. By now, relative motions have been measured only for 258 binaries. 
That is why inferences made from the observations available at this stage should be regarded as preliminary.

I would like to dwe11 on two problems connected closely with the virial mass interpretation of double galaxies: the radial velocity measurement errors and the presence of optical pairs in the sample.

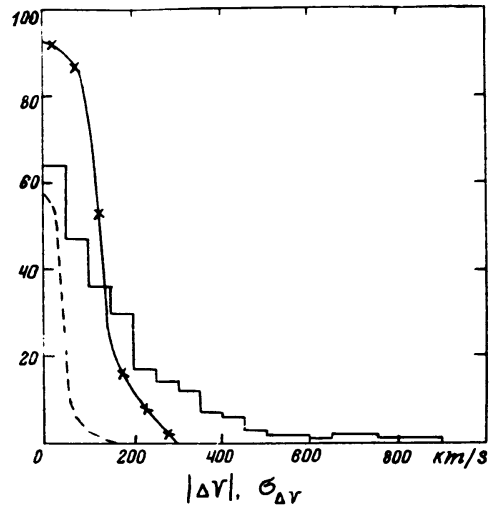

Figure 1. The distribution of radial velocity difference for 258 double galaxies. The solid line and the dashed line show the distribution of measurement errors.

In Figure 1 is presented the distribution of the radial velocity difference for 258 double galaxies. Histogram of the $|\Delta \mathrm{V}|$-distribution has a maximum near zero and also an extended tail up to $1000 \mathrm{~km} / \mathrm{s}$. The solid line drawn through the crosses shows the distribution of measurement errors, $\sigma_{\Delta V}$. As can be seen the radial velocity differences for many pairs are comparable with the errors of measurement. Because $|\Delta V|$ does not exceed $100 \mathrm{~km} / \mathrm{s}$ for half of the sample, to obtain reliable information on the kinematics of double galaxies one evidently needs higher accuracy for the radial velocities. The dashed 1 ine in Figure 1 shows the distribution of errors for 63 pairs observed with the 6-metre telescope using the dispersion $\sim 90 \AA / \mathrm{mm}$. As the analysis shows, it is highly desirable to repeat observations of some pairs with higher dispersion.

For a pair of galaxies with an integral luminosity L, projected distance between components $X$, and an orbital eccentricity $e$, the unbiased estimate of the orbital mass-to-luminosity ratio is

$$
\mathrm{f}_{\mathrm{c}} \equiv \mathrm{M} / \mathrm{L}=32 / 3 \pi\left(1-2 \mathrm{e}^{2 / 3}\right)^{-1} \gamma^{-1} \mathrm{X}\left(\Delta \mathrm{V}^{2}-\sigma_{\Delta \mathrm{V}}^{2}\right) \mathrm{L}^{-1} \text {, }
$$

where $\gamma$ is the gravitational constant, and the numerical coefficient reflects the supposition of an isotropic orientation of double galaxies. For definiteness let us assume that the pair's components have strictly circular motions $(e=0)$, in which case the f-estimates wil1 be a minimum.

The integral distribution of $f_{c}$ for 258 pairs is presented in Figure 2 by dots. A logarithmic scale has been chosen to show the wide range of $f_{c}$ observed. To show the role of radial velocity errors, open 


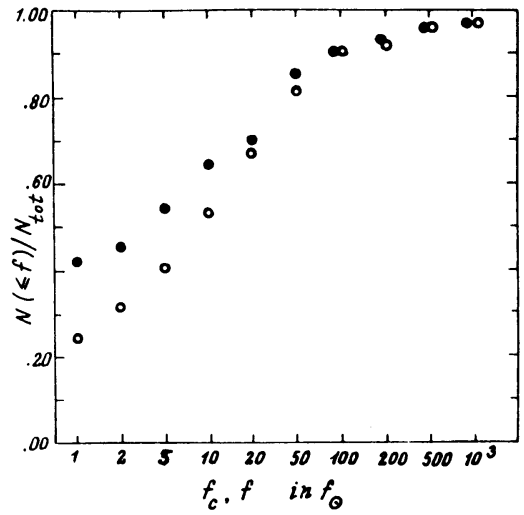

Figure 2. The integral distribution of the mass-to-luminosity ratio for 258 pairs. Dots mark the unbiased estimates; open circles denote the biased estimates.

circles mark the distribution of the biased estimate $f$, calculated for $\sigma_{\Delta V}=0$. From these data it can be seen that more than half of the pairs have $f_{c}<5 f_{\odot}$; however for $10 \%$ of the pairs the mass-toluminosity radio exceeds $100 \mathrm{f}_{\odot}$. The interpretation of large values $\mathrm{f} \simeq\left(10^{2} \div 10^{5}\right) \mathrm{f}_{\odot}$ is apparently connected with the presence of accidental optical pairs in the sample.

Table 1

\begin{tabular}{l|c|ccc|ccc}
\hline \multirow{2}{*}{$\begin{array}{l}\text { Sample of } \\
\text { CPG-pairs }\end{array}$} & $\mathrm{n}_{\text {tot }}$ & \multicolumn{2}{|c|}{$\mathrm{f}<10^{3} \mathrm{f}_{\odot}$} & \multicolumn{2}{|c}{$\mathrm{f}<10^{2} \mathrm{f}_{\odot}$} \\
\cline { 3 - 7 } & 151 & 45 & 33 & 149 & 19 & 8 & 137 \\
\hline $\begin{array}{l}\text { Data by different } \\
\text { authors }\end{array}$ & 43 & 18 & 9 & 42 & 18 & 9 & 42 \\
$\begin{array}{l}\text { Common with } \\
\text { Turner's sample }\end{array}$ & 44 & 39 & 31 & 41 & 12 & 9 & 37 \\
$\begin{array}{l}\text { Observed with the } \\
\text { 5-metre telescope }\end{array}$ & 63 & 16 & 14 & 59 & 10 & 8 & 58 \\
$\begin{array}{l}\text { Observed with the } \\
\text { 6-metre telescope }\end{array}$ & 258 & 37 & 28 & 249 & 16 & 8 & 232 \\
\hline \begin{tabular}{l} 
Total \\
\hline
\end{tabular}
\end{tabular}

Let us consider the inferences made from the data of Table 1 . In this table are presented the mean mass-to-luminosity ratios for different samples of double galaxies from the Catalogue. The first line collects together data based on published estimates of radial velocities, often the radial velocities of different components for one pair being measured by different authors. The second line contains the data for 43 pairs in the Catalogue common with Turner's (1976) sample. In the following lines are presented the results of recent 5-metre telescope 
observations (Karachentsev, Sargent and Zimmermann 1977) and those obtained with the 6-metre telescope (Karachentsev 1977). Alongside the unbiased means $\left\langle\mathrm{f}_{\mathrm{c}}\right\rangle$, in the table there are presented the biased ones, $<\mathrm{f}>$ assuming $\sigma_{\Delta \mathrm{V}}=0$. The left part of the table contains the means calculated after exclusion of undoubted optical pairs with $\mathrm{f}_{\mathrm{C}}>10^{3} \mathrm{f}_{\odot}$; for the right part of the table the optical pair criterion has been taken to be $\mathrm{f}_{\mathrm{c}}>10^{2} \mathrm{f}_{\mathrm{o}}$.

From these data the dependence is seen of the mean orbital mass-toluminosity ratio on both the accuracy of the radial velocity measurements and the presence of optical pairs in the sample. Comparison of the last column data shows that irrespective of the sample the mean values, $\left\langle\mathrm{f}_{\mathrm{c}}\right\rangle=(8 \div 9) \mathrm{f}_{\odot}$, are practically the same and correspond to normal mass-to-luminosity ratios in the absence of massive coronae.

The role of optical pairs in the interpretation of <f>-values has been repeatedly emphasized by Fessenko (1976). According to preliminary calculations the criterion for isolated pairs used in the Catalogue allows for the presence in the sample of about $10 \%$ of the optical pairs. This agrees quantitatively with the supposition that all 26 pairs, having $\mathrm{f}_{c}>100 \mathrm{f}_{\odot}$, out of the total number 258 , are optical pairs. The correlation of apparent magnitudes and radial velocities for these pairs gives further evidence for this (see Figure 3). The members of each pair are marked in the figure by dots linked by straight lines and the dashed lines denote a $90 \%$ confidence interval for a distribution of field galaxies relative to mean dependence $\mathrm{m} \sim 51 \mathrm{ogV}$. As expected for optical pairs their vectors are oriented mainly along Hubble's relation.

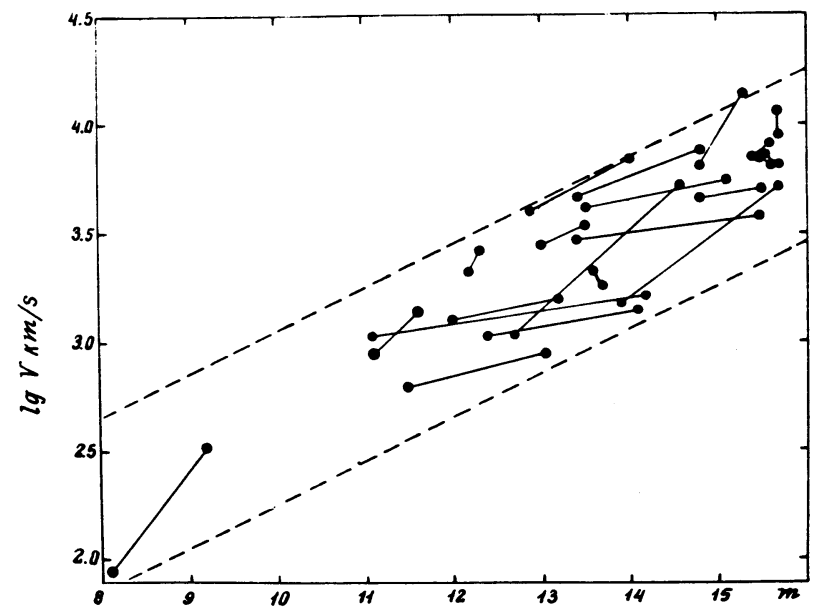

Figure 3. The correlation of apparent magnitudes and radial velocities for optical pairs.

Considering double galaxies for the components of which radial velocity differences have been measured repeatedly by different authors, one finds rather a large number of cases in which the $\Delta \mathrm{V}-$ estimates are poorly reproduced. For instance, according to RCG the 
velocity difference for the interacting pair of spirals CPG $295=$ VV 228 $=\operatorname{Arp} 294$ is $\Delta \mathrm{V}=(410 \pm 148) \mathrm{km} / \mathrm{s}$. New measurements give the value $\Delta \mathrm{V}=(60 \pm 22) \mathrm{km} / \mathrm{s}$, which reduces the former high estimate $\mathrm{f}_{\mathrm{c}}=77 \mathrm{f}_{\odot}$ to a normal value, $1.7 \mathrm{f}_{\odot}$, for spiral galaxies.

There exist a number of effects which may lead to noticeable errors in measuring radial velocities. Here are some of them:

(1) Blending of absorption lines in galaxy spectra by the nightsky lines (Simkin 1977).

(2) A possible non-coincidence of the mean velocity of a galaxy measured from emission and from absorption lines when the object has large non-circular internal motions.

(3) A composite structure of a number of tight interacting pairs in which no distinct nucleus is observed as the dynamical centre of the components. Examples of such pairs have been given by Tifft (1977). Spectra obtained with scale 8.'6 per mm at the slit show that the splitting of the emission lines is caused by discrete structure of the emitting clouds.

(4) Some residual effects of image-tube distortion.

(5) Different estimates by different authors of the radial velocity corrections due to the motion of the Sun and the Earth.

These errors may lead to a total value $\sigma_{\mathrm{V}} \simeq 50 \mathrm{~km} / \mathrm{s}$, and may lead to systematic overestimation of virial masses, especially for wide pairs. So, the error in velocity of each component $\sim 50 \mathrm{~km} / \mathrm{s}$ gives a fictitious value $\mathrm{f} \simeq 80 \mathrm{f}_{\odot}$ for a pair having $\mathrm{L}=1 \times 10^{10} \mathrm{~L}_{\odot}$ and $\mathrm{X}=200 \mathrm{kpc}$. Obviously, if these effects are ignored, evidence may be found for unobservable coronae in double galaxies.

\section{SMALL GROUPS OF GALAXIES}

The exclusion of optical members in groups of galaxies is a more difficult task in comparison with pairs. There are systems, for example, Stephan's Quintet and Seyfert's Sextet, where the radial velocities of one or two members differ considerably from the rest. This leads to a large M/L - value for a system. Exclusion of such members reduces the virial mass estimate by a large factor.

As another similar example I present the sextet of galaxies in Cetus in which there is a large redshift difference. This system has been studied by Karachentsev and Tifft (1977). A reproduction of this group from the Palomar Sky Survey print is presented in Figure 4. The radial velocities of galaxies are marked. As can be seen, the radial velocities of the two northern galaxies ( $C$ and $D$ ) differ from the rest by $\sim 1000 \mathrm{~km} / \mathrm{s}$. The dynamical parameters for the group are shown in Table 2. The notations of values in it are generally accepted. From data in the table it follows that the hypothesis of accidental projec- 


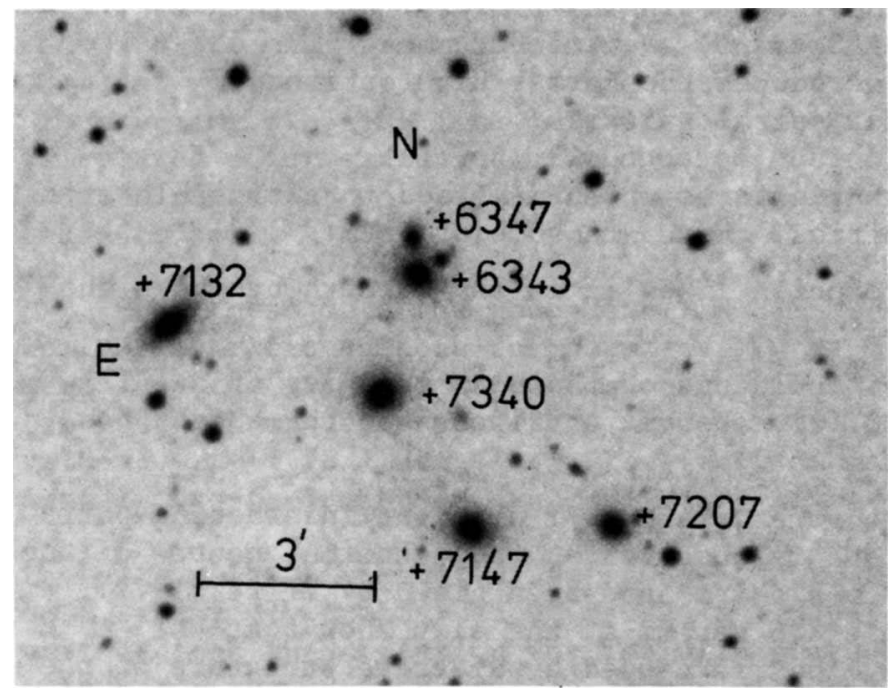

Figure 4. The group of galaxies in Cetus with a large redshift difference. The reproduction is from the Palomar Sky Survey print. Radial velocities of the galaxies are marked.

Table 2

\begin{tabular}{ccccccc}
\hline Combination & $\begin{array}{c}\left\langle\mathrm{V}_{\mathrm{o}}\right\rangle \\
\mathrm{km} / \mathrm{s}\end{array}$ & $\begin{array}{c}\left\langle\mathrm{V}_{\mathrm{r}}^{2}\right\rangle^{\frac{1}{2}} \\
\mathrm{~km} / \mathrm{s}\end{array}$ & $\begin{array}{c}\left\langle\mathrm{R}_{\mathrm{i} j}^{-1}\right\rangle^{-1} \\
\mathrm{kpc}\end{array}$ & $\begin{array}{c}\mathrm{L} \\
\mathrm{L}_{\odot}\end{array}$ & $\begin{array}{c}\mathrm{f} \\
\mathrm{f}_{\odot}\end{array}$ & $\begin{array}{c}\mathrm{f}_{\mathrm{c}} \\
\mathrm{f}_{\odot}\end{array}$ \\
\hline $\mathrm{A}+\mathrm{B}+\mathrm{C}+\mathrm{D}+\mathrm{E}+\mathrm{F}$ & +6919 & 411 & 70.8 & $3.7210^{10}$ & 848 & 835 \\
$\mathrm{~A}+\mathrm{B}+\mathrm{E}+\mathrm{F}$ & +7206 & 82 & 99.8 & $3.4710^{10}$ & 56 & 36 \\
$\mathrm{C}+\mathrm{D}$ & +6345 & 2 & 14.1 & $0.5410^{10}$ & 0.03 & - \\
\hline
\end{tabular}

tion of the pair ( $C$ and $D$ ) on the quartet (A,B,E,F) eliminates the virial paradox. In spite of the fact that the group in Cetus is apparently isolated the mathematical expectation of a number of optical configurations similar to it is $n \gtrsim 1$ among galaxies contained in Zwicky's catalogue.

The presence of interacting features in groups of galaxies reduces considerably the probability of finding optical members in them. A rich collection of such systems is included in the two parts of the "Atlas of Interacting Galaxies" by Vorontsov-Velyaminov $(1959,1977)$. The Moscow State Institute of Astronomy in cooperation with Special Astrophysical Observatory has started a programme of spectral investigation of different types of interacting systems with the 6-metre telescope. Examples of spectrograms of several VV-objects are presented in Figure 5. From the results of the first observations (Arkhipova et al. 1977) the following parameters have been estimated for 23 interacting 


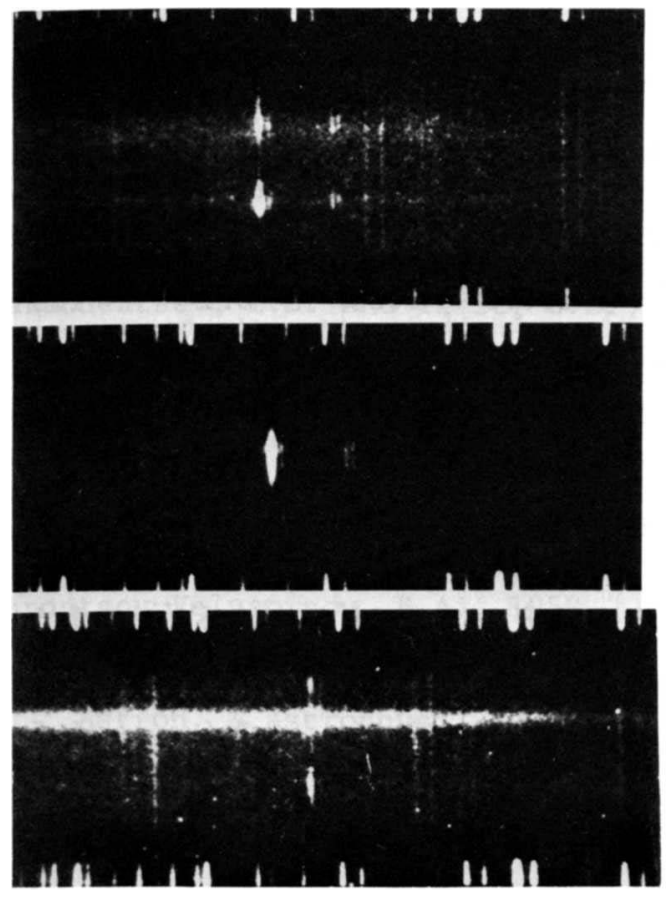

Figure 5. The spectrograms of interacting systems: (a) VV 620, (b) VV 394, (c) VV 615. The original dispersion is $\sim 90 \AA / \mathrm{mm}$ and the scale on the slit is $8 . ' 6$ per $\mathrm{mm}$.

systems classified by Vorontsov-Velyaminov as "nests", "minichains", etc., - radial velocities, $\left\langle\Delta V_{r}^{2}>\frac{1}{2}\right.$, the effective radius $R$, the virial mass $\mathrm{M}$, and the luminosity $\mathrm{L}$. The basic data for these systems are presented in Table 3 . The mean virial mass-to-luminosity ratio, $\left\langle\mathrm{f}_{\mathrm{c}}\right\rangle=(6.9 \pm 2.9) \mathrm{f}_{\Theta}$, with $\mathrm{H}=75 \mathrm{~km} / \mathrm{s} \mathrm{Mpc}$, shows that in the sample optical members are practically absent. For a wide range of sizes $(0.2 \mathrm{kpc} \div 30 \mathrm{kpc})$ and luminosities $\left(6 \times 10^{7}-1 \times 10^{11}\right) \mathrm{L}_{\odot}$ of interacting systems their mass-to-luminosity ratios are approximately the same.

\section{CONCLUSION}

The data presented show that close interacting systems, and also pairs of galaxies both tight and wide have on the average a normal virial mass-to-luminosity ratio $\left\langle\mathrm{f}_{\mathrm{c}}\right\rangle \simeq 8 \mathrm{f}_{\Theta}$, which can be $\exp 1$ ained without the hypothesis of the existence of massive hidden coronas around galaxies. 
Table 3

\begin{tabular}{|c|c|c|c|c|c|c|c|c|}
\hline$n^{0}$ & VV & $\begin{array}{l}<\mathrm{V}_{\mathrm{o}}> \\
\mathrm{km} / \mathrm{s}\end{array}$ & $\begin{array}{c}<\Delta \mathrm{V}_{\mathrm{r}}^{2}>^{\frac{1}{2}} \pm \sigma_{\Delta \mathrm{V}} \\
\mathrm{km} / \mathrm{s}\end{array}$ & $\begin{array}{c}\mathrm{R} \\
\mathrm{kpc}\end{array}$ & $\begin{array}{l}\mathrm{M} / 10^{10} \\
\mathrm{M}_{\Theta}\end{array}$ & $\begin{array}{l}\mathrm{L} / 10^{10} \\
\mathrm{~L}_{\Theta}\end{array}$ & $\begin{array}{l}\mathrm{f} \\
\mathrm{f}_{\odot}\end{array}$ & $\begin{array}{l}f_{c} \\
f_{o}\end{array}$ \\
\hline 1 & 587 & 1808 & $6 \pm 23$ & 27.4 & 0.078 & 0.83 & 0.1 & -1.3 \\
\hline 2 & $587 \mathrm{E}$ & 1805 & $\leqslant 65$ & 1.0 & 0.97 & 0.11 & & $\leqslant 8.8$ \\
\hline 3 & 555 & 4320 & $39 \pm 20$ & 7.5 & 3.1 & 0.52 & 5.9 & 4.4 \\
\hline 4 & 699 & 4407 & $31 \pm 20$ & 30.8 & 9.7 & 2.5 & 3.8 & 2.2 \\
\hline 5 & 644 & 3714 & $123 \pm 20$ & 3.0 & 2.9 & 1.7 & 1.7 & 1.6 \\
\hline 6 & 141 & 2751 & $46 \pm 30$ & 4.8 & 0.8 & 0.31 & 2.6 & 1.5 \\
\hline 7 & 761 & 12024 & $71 \pm 35$ & 26.6 & 44.0 & 4.05 & 10.9 & 8.3 \\
\hline 8 & 541 & 2568 & $75 \pm 20$ & 7.9 & 3.5 & 2.16 & 1.6 & 1.5 \\
\hline 9 & 620 & 1659 & $52 \pm 44$ & 17.7 & 3.8 & 0.63 & 6.0 & 1.7 \\
\hline 10 & 794 & 575 & $\leqslant 20$ & 2.2 & 0.19 & 0.010 & & $\leqslant 19$ \\
\hline 11 & 498 & 1319 & $23 \pm 20$ & 1.8 & 0.31 & 0.059 & 5.2 & 1.4 \\
\hline 12 & 457 & 1292 & $28 \pm 20$ & 2.0 & 0.34 & 0.14 & 2.4 & 1.2 \\
\hline 13 & 126 & 10427 & $57 \pm 30$ & 25.1 & 6.4 & 11.1 & 0.6 & 0.4 \\
\hline 14 & 523 & 3100 & $116 \pm 25$ & 4.9 & 5.3 & 1.1 & 4.8 & 4.6 \\
\hline 15 & 497 & 888 & $\leqslant 20$ & 2.8 & 0.25 & 0.027 & & $\leqslant 9.3$ \\
\hline 16 & 261 & 2583 & $6 \pm 30$ & 3.2 & 0.01 & 1.42 & 0.0 & -0.2 \\
\hline 17 & 432 & -245 & $79 \pm 30$ & 2.3 & 1.14 & 0.068 & 16.8 & 12.4 \\
\hline 18 & 558 & 188 & $\leqslant 30$ & 1.9 & 0.034 & 0.024 & & $\leqslant 1.4$ \\
\hline 19 & 48 & 3570 & $34 \pm 26$ & 26.4 & 2.18 & 5.44 & 0.4 & 0.2 \\
\hline 20 & 339 & 7253 & $240 \pm 20$ & 38.3 & 174. & 2.86 & 60.9 & 60.5 \\
\hline 21 & 394 & 438 & $\leqslant 15$ & 0.2 & 0.010 & 0.0065 & & $\leqslant 1.5$ \\
\hline 22 & $\begin{array}{l}394+ \\
\text { M101 }\end{array}$ & 426 & $23 \pm 15$ & 19.0 & 0.79 & 2.36 & 0.3 & 0.2 \\
\hline 23 & 615 & 2516 & $93 \pm 20$ & 4.0 & 11.4 & 0.63 & 18.1 & 17.2 \\
\hline
\end{tabular}




\section{REFERENCES}

Arkhipova, V.P., Afanas'ev, V.L., Dosta1, V.A., Karachentsev, I.D. and Metlov, V., 1977 (in press).

Fessenko, B.I., 1976. Soviet Astron. Zh., 53, 112.

Fisher, J.R. and Tully, R.B., 1975. Astron. \& Astrophys., 44, 151.

Karachentsev, I.D., 1972. Catalogue of Isolated Pairs of Galaxies in Northern Hemisphere, Soobsch. Special Astrophys. Obs., 7, 3 .

Karachentsev, I.D. and Tifft, W.G., 1977. Astron. \& Astrophȳs. (in press).

Karachentsev, I.D., Sargent, W.L.W. and Zimmermann, B., 1977 (in press). Karachentsev, I.D., 1977 (in press).

Materne, J. and Tammann, G.A., 1974. Astron. \& Astrophys., 37, 383.

Simkin, S.M., 1977. Astron. \& Astrophys., 55, 369.

Tifft, W.G., 1977. Astrophys.J., 211, 31 .

Turner, E.L., 1976. Astrophys. J., 208, 20.

Vorontsov-Velyaminov, B.A., 1959. Atlas and Catalogue of Interacting Galaxies, I, Moscow State Univ., Moscow.

Vorontsov-Velyaminov, B.A., 1977. Atlas of Interacting Galaxies, Astron. \& Astrophys., Supp1., 28, 1 .

\section{DISCUSSION}

Zeldovich: What is the value of $\mathrm{M} / \mathrm{L}$ for our own galaxy?

Karachentsev: About 5 to $10 \mathrm{f}_{\odot}$.

Ostriker: What is the mean projected separation of your large sample of binary galaxies?

Karachentsev: $30 \mathrm{kpc}$ assuming a Hubble constant $\mathrm{H}=75 \mathrm{~km} \mathrm{~s}^{-1} \mathrm{Mpc}^{-1}$.

Ostriker: In that case the mass you find $[(M / L) \times L]$ is just what would be expected, even if there were a great deal of mass at distances of $100-1000 \mathrm{kpc}$ from the galaxy centres.

Karachentsev: But I find the values of $\mathrm{M} / \mathrm{L}$ to be independent of the separation of the galaxies including those with separations $>100 \mathrm{kpc}$.

Zasov: Is there any difference between the values of M/L for pairs of elliptical and pairs of spiral galaxies?

Karachentsev: Yes. For pairs of ellipticals M/L is about twice as large as it is for spiral pairs.

Audouze: What is the value of the declaration parameter that you deduce or favour from your analysis? I guess that it is a very small one.

Karachentsev: The low mass-to-luminosity ratio for pairs and tight groups does not contradict any value of $\mathrm{q}_{\mathrm{o}}$ in the range $0.01-0.2$ because 
the bulk of the hidden virial matter may be associated with much richer systems .

Jaakkola: The virial mass problem is dependent on the real nature of redshifts. Specifically, I refer to the usual practice of assuming that a positive magnitude-redshift relation for possible members of double systems, groups and clusters is taken as evidence for these systems being chance projection. However, such a relation is also expected starting from the overall phenomenology of the non-velocity redshifts. For example, if associations between some quasars and bright galaxies are physical ones, there is an intrinsic $(\mathrm{m}, \mathrm{z})$-relation in the system. Such relations appear to be a common effect in systems of galaxies (Jaakkola, Proc. III European Meeting, Tbilisi 1975, p.218). The effect is also present in pairs and groups of Zwicky's galaxies (Jaakkola, Astrophys. Space Sci. 49, 99, 1977). In the 1atter systems, there are in most cases evidence of physical interactions and hence the relation is not due to projection effects. 